Correspondence

Anil K. Tripathi

tripathianil@rediffmail.com

Received 3 January 2008

Revised 14 March 2008

Accepted 15 March 2008

\section{An extra-cytoplasmic function sigma factor and anti-sigma factor control carotenoid biosynthesis in Azospirillum brasilense}

\author{
Nagarajan Thirunavukkarasu, ${ }^{1}$ Mukti Nath Mishra, ${ }^{1}$ Stijn Spaepen, ${ }^{2}$ \\ Jos Vanderleyden, ${ }^{2}$ Carol A. Gross ${ }^{3}$ and Anil K. Tripathi ${ }^{1}$ \\ ${ }^{1}$ School of Biotechnology, Faculty of Science, Banaras Hindu University, Varanasi-221005, India
${ }^{2}$ Centre of Microbial and Plant Genetics, Department of Microbial and Molecular Systems, Faculty of
Bioscience Engineering, Katholieke Universiteit Leuven, Kasteelpark Arenberg 20, B-3001
Heverlee, Belgium
${ }^{3}$ Departments of Microbiology and Immunology, and Cell and Tissue Biology, University of
California, San Francisco, CA 94158-2517, USA
}

Strains Sp7 and Cd of Azospirillum brasilense, a plant growth-promoting rhizobacterium, differ in synthesis of carotenoids. While colonies of strain Sp7 have a white-cream colour on plates, colonies of strain $\mathrm{Cd}$ are orange-pink coloured because of the synthesis of carotenoids.

Screening of a mini-Tn5 mutant library of $A$. brasilense Sp7 revealed two orange-pink-coloured mutants that produced carotenoids. Cloning and sequencing of the Tn5 flanking region in both the carotenoid-producing mutants of Sp7 revealed insertion of Tn5 in an ORF encoding anti- $\sigma$ factor, a ChrR-like protein. The upstream region of the Tn5-mutated ORF contained another ORF that encoded an extra-cytoplasmic function (ECF)-class $\sigma$ factor ( $\left.\sigma^{\mathrm{E}}, \mathrm{RpoE}\right)$. When the nucleotide sequences of the corresponding ORFs from the carotenoid-producing strain $\mathrm{Cd}$ were analysed, the sequence of the $\mathrm{Cd} \sigma^{\mathrm{E}}$ was identical to that of the carotenoid non-producing strain Sp7, but the Cd anti- $\sigma^{\mathrm{E}}$ ORF had a deletion that caused frame shifting and creation of a stop codon. This resulted in the premature termination of the protein, which was about $7 \mathrm{kDa}$ smaller than the Sp7 anti- $\sigma^{\mathrm{E}}$. Cloning of Sp7 anti- $\sigma^{\mathrm{E}}$ in a broad-host-range expression vector and expression in $A$. brasilense $\mathrm{Cd}$ and in the anti- $\sigma^{\mathrm{E}}$ knockout mutant of $A$. brasilense $\mathrm{Sp} 7$ resulted in the inhibition of carotenoid synthesis. Similarly, cloning and overexpression of $A$. brasilense Sp7 $\sigma^{\mathrm{E}}$ in $A$. brasilense Sp7 resulted in the production of carotenoids. These observations clearly indicate that carotenoid synthesis in $A$. brasilense is controlled by $\sigma^{\mathrm{E}}$ with its cognate anti- $\sigma^{\mathrm{E}}$.

\section{INTRODUCTION}

Members of the genus Azospirillum are nitrogen-fixing bacteria that live in close association with the roots of several important crop plants and grasses, and stimulate the growth of their host plant by producing phytohormones and siderophores (Steenhoudt \& Vanderleyden, 2000). Taxonomically, they are closely related to Rhodospirillum rubrum and Magnetospirillum magnetotacticum of

Abbreviation: ECF, extra-cytoplasmic function.

The GenBank/EMBL/DDBJ accession numbers for the $\sigma^{\mathrm{E}}$ and anti- $\sigma^{\mathrm{E}}$ sequences of $A$. brasilense are D0324853 and DO324854, respectively.

Three supplementary figures showing phylogenetic trees for the amino acid sequences of ChrR and ECF- $\sigma^{\mathrm{E}}$, and the effects of the expression of rpoE and chrR in Azospirillum brasilense, are available with the online version of this paper. the family Rhodospirillaceae (Woese et al., 1982). Currently, the genus consists of 10 species, including Azospirillum brasilense, Azospirillum lipoferum, Azospirillum halopraeferens, Azospirillum amazonense, Azospirillum irakense, Azospirillum largimobile, Azospirillum doebereinerae, Azospirillum oryzae, Azospirillum melinis and Azospirillum canadense (Mehnaz et al., 2007). Some of the strains of $A$. brasilense and A. lipoferum show orange-pink pigmentation due to the presence of carotenoids (Tarrand et al., 1978). Within A. brasilense, two strains (Cd and Sp7) differ from each other with regard to their carotenoid content. While Cd produces carotenoids, giving an orange-pink colour to the colonies, Sp7 lacks detectable levels of carotenoids (Tarrand et al., 1978; Nur et al., 1981). Strains Sp7 and Cd are genetically very closely related. It has even been suggested that strain $\mathrm{Cd}$ is a reisolate following plant inoculation with $\mathrm{Sp} 7$ (Nur et al., 1981). 
Although A. brasilense Sp7 is not reported to produce carotenoids, carotenoid-producing mutants (spontaneous or induced by chemical mutagens or Tn5) of this strain have been isolated (Tarrand et al., 1978; Abdel-Salam \& Klingmueller, 1987). The synthesis of carotenoids in these mutants is triggered by oxygen stress (Hartmann \& Hurek, 1988), and their presence has been shown to improve oxygen tolerance for nitrogen fixation at high dissolved oxygen concentrations $(12 \mu \mathrm{M})$ compared with their pigmentless parent.

Besides the enzymes that detoxify superoxide and hydrogen peroxide, carotenoids in $A$. brasilense strain $\mathrm{Cd}$ are implicated in protection against oxidative damage owing to their ability to quench singlet oxygen and possibly oxygen radicals (Hartmann \& Hurek, 1988; Nur et al., 1981, 1982). The fact that nitrogen fixation is inhibited in A. brasilense $\mathrm{Cd}$ at high dissolved oxygen concentrations upon inhibition of carotenoid synthesis by diphenylamine suggests that carotenoids protect nitrogenase from oxidative damage (Nur et al., 1981).

On the basis of similarity to the absorption spectra of carotenoids from Halobacterium cutirubrum, the carotenoids of $A$. brasilense Sp7 mutants and those present in the A. brasilense $\mathrm{Cd}$ strain were thought to be of the bacterioruberin type (Nur et al., 1981). The long-chain C-50 bacterioruberin-type carotenoids have been isolated from several extremophilic bacteria and Archaea, including species of Halobacterium, Haloferax (D'Souza et al., 1997) and psychrotrophic bacteria [Micrococcus roseus (Jagannadham et al., 1991) and Arthrobacter agilis (Fong et al., 2001)]. These organisms require adaptive mechanisms to cope with extreme environmental conditions, and bacterioruberin has been shown to contribute to their adaptation to and survival in extreme environments. The presence of these carotenoids may also reinforce the membrane bilayer to reduce $\mathrm{O}_{2}$ diffusion in the cytoplasm (Wisniewska \& Subczynski et al., 1998) and raise the hydrophobic barrier for polar molecules and ions.

While screening a mini-Tn 5 mutant library of $A$. brasilense Sp7, two mutants, Car-1 and Car-2, that were orange-pink coloured due to the production of carotenoids, were identified. Activation of the synthesis of carotenoids in the two Tn5 mutants suggested to us that a repressor of carotenoid biosynthesis had been inactivated in these strains. Although carotenoid-producing mutants of $A$. brasilense Sp7 have been isolated and their phenotype analysed, the genetic basis of carotenoid production has not been investigated (Hartmann \& Hurek, 1988). In this study we have shown that inactivation of the genetic locus encoding anti- $\sigma$ factor in $A$. brasilense Sp7 leads to carotenoid production in the mutants. The occurrence of a deletion in the ORF of the gene encoding anti- $\sigma$ factor in a naturally carotenoid-producing strain, A. brasilense $\mathrm{Cd}$, further corroborated the role of an extra-cytoplasmic function $\sigma$ factor and its cognate anti- $\sigma$ factor in controlling carotenoid production in A. brasilense.

\section{METHODS}

Bacterial strains, plasmids, chemicals and growth conditions. A. brasilense strains were grown in minimal medium for A. brasilense (MMAB; Vanstockem et al., 1987) containing malate and $\mathrm{NH}_{4} \mathrm{Cl}$ $(10 \mathrm{mM})$ as sole source of carbon and nitrogen, respectively. Plasmid pCMPG8003 (Nagarajan et al., 2007) was used to generate random insertions of a transposon (Tn5) in A. brasilense Sp7. Another plasmid, pJDN30 (Newman et al., 1999), containing a fusion of $r p o E$ P1 promoter with $l a c Z$ on a broad-host-range vector, was conjugatively mobilized via Escherichia coli S.17-1 into A. brasilense Sp7 and the Car-1 mutant (Vanstockem et al., 1987). Assays of $\beta$-galactosidase activity were performed in triplicate as in Newman et al. (2001), except that the cells were grown aerobically with shaking in MMAB medium. All chemicals used for growing bacteria were from Himedia, while methanol (HPLC grade) was from Merck.

Screening of the mutant library, cloning and characterization of Tn5 mutations. A mutant library of A. brasilense Sp7 containing 6800 mutants generated by random insertion of mTn5gusA-oriV Tn 5 was screened visually for orange-pink-coloured colonies on minimal agar plates containing $40 \mu \mathrm{g}$ kanamycin $\mathrm{ml}^{-1}$. Total genomic DNA isolated with the aid of a GFX DNA purification kit (Amersham Pharmacia Biotech) was digested with restriction endonucleases (NdeI or XhoI) that do not cut mTn5gusA-oriV. The presence of oriV in the mTn5 cassette facilitated direct cloning by ligating the restrictionendonuclease-digested genomic DNA using T4 DNA ligase (Invitrogen) without a cloning vector. The NdeI fragment containing an $\operatorname{mTn} 5$ insertion in the genomic region of the Car- 1 mutant was self-ligated and transformed into E. coli $\mathrm{DH} 5 \alpha$, and the resulting plasmid was designated pTN1. A partial sequence of the genomic DNA region flanking both sides of the mTn5 insertion was determined by using the primers $5^{\prime}$-CTCCTTAGCTAGTCAGGTACCG-3', which binds close to the NotI site of gusA, and 5'GTCTGACGCTCAGTGGAAC-3', which binds in the oriV region of mTn5. DNA sequence similarities were examined using the BLASTX program at NCBI (Altschul et al., 1997). The CLUSTAL w program (Thompson et al., 1994) was used to align the target sequences with similar sequences retrieved from the databases, and a dendrogram was constructed using the neighbour-joining method (Saitou \& Nei, 1987) with the program MEGA version 3.0 (Kumar et al., 2004). The robustness of the inferred trees was evaluated by 500 bootstrap resamplings.

Cloning of rpoE and chrR genes in the expression vector pET15b. PCR amplified $r p o E$ and $c h r R$ genes were cloned in the expression vector, pET15b digested with $\mathrm{NdeI}$ and BamHI. The rpoE gene was PCR-amplified by using the primers rpoE2-FNde ( $5^{\prime}$ GGAATTCCATATGCAGGATCTTGGTTCCCC-3'), with a NdeI site located upstream of the start codon, and rpoE-RBam (5'CGGGATCCTCATCGGGAGTCCCTCATG-3'), with a BamHI site located downstream of the stop codon (restriction sites are underlined). Similarly, the $c h r R$ gene was amplified by using the primers arpoE-FNde (5'-ACTCCCGCATATGACCGTGCCCACCCACC-3') and $c h r R$-RBam (5'-CGGGATCCTCAGAACTTCACAAACGGGTTGAGG-3'). The amplicons were digested with NdeI and BamHI, PCR-purified and ligated with the similarly digested expression vector pET15b. E. coli $\mathrm{DH} 5 \alpha$ was transformed with the ligation mix and transformants were selected on Luria agar containing $100 \mu \mathrm{g}$ ampicillin $\mathrm{ml}^{-1}$. The nucleotide sequences of the clones of $r p o E$ and $c h r R$ were determined, and showed the expected size of inserts.

pET15b clones harbouring rpoE and arpoE genes from A. brasilense Sp7 and A. brasilense Cd were designated pAT1and pAT2 (rpoE), and pAT3 and pAT4 (chrR), respectively (see Table 1). These clones were then transferred into E. coli BL21 2 DE3 (pLysS). The clones were grown overnight in LB medium with $25 \mu \mathrm{g}$ chloramphenicol $\mathrm{ml}^{-1}$ 
Table 1. Bacterial strains and plasmids used

\begin{tabular}{|c|c|c|}
\hline Strain or plasmid & Relevant properties & Source or reference \\
\hline \multicolumn{3}{|l|}{ Strains } \\
\hline E. coli $\mathrm{DH} 5 \alpha$ & $\Delta l a c U 169$ hsdR17 recA1 endA1 gyrA96 thiL relA1 & Gibco-BRL \\
\hline E. coli S17-1 & $\mathrm{Sm}^{\mathrm{r}}$, recA, thi, pro, hsdR ${ }^{-} \mathrm{M}+\mathrm{RP} 4: 2-\mathrm{Tc}: \mathrm{Mu}: \mathrm{Km} \mathrm{Tn} 7, \lambda$ pir & Nagarajan et al. (2007) \\
\hline E. coli BL21 $\lambda$ DE3(pLysS) & $o m p T h s d S\left(r_{B}^{-} m_{B}^{-}\right) \mathrm{dcm}+\operatorname{Tet}^{\mathrm{r}}$ endA gald(DE3) & Novagen \\
\hline A. brasilense $\mathrm{Sp} 7$ & Carotenoid-non-producing strain & Nur et al. (1981) \\
\hline A. brasilense $\mathrm{Cd}$ & Carotenoid-producing strain & Nur et al. (1981) \\
\hline Car-1 & Carotenoid-producing A. brasilense $\mathrm{Sp} 7$ mutant with $\mathrm{mTn} 5$ insertion & This work \\
\hline Car-2 & Carotenoid-producing A. brasilense $\mathrm{Sp} 7$ mutant with $\mathrm{mTn} 5$ insertion & This work \\
\hline \multicolumn{3}{|c|}{${ }^{2}$} \\
\hline pCMPG8003 & $\mathrm{Ap}^{\mathrm{r}} \mathrm{Km}^{\mathrm{r}}, \mathrm{mTn} 5 g u s A$-oriV in pUT vector & Nagarajan et al. (2007) \\
\hline pET15b T7 & Expression vector & Novagen \\
\hline pTN1 & $\begin{array}{l}\text { Self-ligated NdeI fragment containing } \mathrm{mTn} 5 \mathrm{OriV} \text { and flanking genomic region from } \\
\text { Car-1 }\end{array}$ & This work \\
\hline pMMB206 & $\mathrm{Cm}^{\mathrm{r}}$, broad-host-range low-copy-number expression vector & Morales et al. (1991) \\
\hline pAT1 & rpoE gene from A. brasilense Sp7 cloned into NdeI and BamHI sites of pET15b & This work \\
\hline pAT2 & rpoE gene from A. brasilense Cd cloned into NdeI and BamHI sites of pET15b & This work \\
\hline pAT3 & chrR gene from A. brasilense Sp7 cloned into NdeI and BamHI sites of pET15b & This work \\
\hline pAT4 & chrR gene from A. brasilense Cd cloned into NdeI and BamHI sites of pET15b & This work \\
\hline pAT5 & rpoE gene from A. brasilense Sp7 cloned into EcoRI and BamHI sites of pMMB206 & This work \\
\hline pAT6 & chrR gene from A. brasilense Sp7 cloned into EcoRI and BamHI sites of pMMB206 & This work \\
\hline pJDN30 & rpoE $\mathrm{P} 1$ promoter of $R$. sphaeroides cloned into lacZ fusion reporter vector pRKK200 & Newman et al. (1999) \\
\hline
\end{tabular}

and $100 \mu \mathrm{g}$ ampicillin $\mathrm{ml}^{-1}$, and inoculated into fresh medium so as to give an $\mathrm{OD}_{600}$ of 0.03 . Cultures were then incubated with shaking at $25{ }^{\circ} \mathrm{C}$ until they attained $\mathrm{OD}_{600} 0.3$. At this stage, IPTG was added to give a final concentration of $1 \mathrm{mM}$ to induce the expression of the cloned gene. Aliquots of the cultures were taken every $30 \mathrm{~min}$, and were mixed with TCA to hydrolyse the cells and kept at $4{ }^{\circ} \mathrm{C}$ to precipitate the proteins. Equal amount of proteins from each sample were loaded and resolved on $15 \%$ SDS-PAGE gels. After having optimized the conditions for induction, a $30 \mathrm{ml}$ culture volume was used to induce the expression of proteins. Cultures induced for $3 \mathrm{~h}$ were pelleted and used for extraction and purification of induced proteins using Ni-NTA resin, as described in the QIAexpress manual.

Cloning of rpoE and chrR genes in the broad-host-range expression vector pMMB206, and conjugative mobilization into A. brasilense. PCR-amplified $r p o E$ and $c h r R$ genes were cloned into the broad-host-range expression vector pMMB206, digested with EcoRI and BamHI. The rpoE gene was PCR-amplified by using the primers rpoE-FEco (5' -GGAATTCAATGCAGGATCTTGGTTCCCC$3^{\prime}$ ), with an EcoRI site (underlined) located upstream of the start codon, and rpoE-RBam (5'-CGGGATCCTCATCGGGAGTCCCTCATG-3'), with a BamHI site (underlined) located downstream of the stop codon. Similarly, the $c h r R$ gene was amplified by using the primers $c h r R$ arpoE-FEco (5'-GGAATTCAATGACCGTGCCCACCCACCATC-3') and arpoE-RBam (5'-CGGGATCCTCAGAACTTCACAAACGGGTTGAGG-3'). The amplicons were digested with EcoRI and BamHI, PCR-purified and ligated with the similarly digested pMMB206. E. coli $\mathrm{DH} 5 \alpha$ was transformed with the ligation mix and transformants were selected by blue/white selection on Luria agar plates containing $1 \mathrm{mM}$ IPTG, $80 \mu \mathrm{g} \mathrm{X}-\mathrm{Gal} \mathrm{m}{ }^{-1}$ and $25 \mu \mathrm{g}$ chloramphenicol ml $\mathrm{m}^{-1}$. Nucleotide sequences of the clones from white colonies harbouring inserts of the expected size were determined. The clones harbouring rpoE (pAT5) and chrR (pAT6) genes from A. brasilense Sp7 were transferred into E. coli S17-1, and then conjugatively mobilized into $A$. brasilense Sp7, the Car-1 mutant and $A$. brasilense $\mathrm{Cd}$, as described above.

Spectrophotometric analysis for carotenoids. The bacterial cultures were allowed to grow to stationary phase with shaking at 150 r.p.m. in an incubator-shaker at $30{ }^{\circ} \mathrm{C}$ in MMAB medium. Cells were then pelleted and washed with saline solution $(0.85 \% \mathrm{NaCl}$ in water). Fresh cells were suspended in methanol repeatedly to extract the pigments whenever required to characterize them by absorption spectra (Jagannadham et al., 1991). Pigment extraction was carried out at room temperature, and all glassware was covered with aluminium foil to protect the pigments from light. UV-visible absorption spectra between 300 and $600 \mathrm{~nm}$ of the pigments dissolved in methanol were recorded with a Hitachi U-2000 spectrophotometer. To study the effects of cloned rpoE and $c h r R, A$. brasilense cultures were grown in $50 \mathrm{ml} \mathrm{LB}$ medium for $24 \mathrm{~h}$ with appropriate antibiotics and IPTG, if required. The growth of the cultures was estimated by recording $\mathrm{OD}_{600}$, after which the cell density was equalized by suitable dilution. Cultures $(40 \mathrm{ml})$ with equal $\mathrm{OD}_{600}$ values were used for the extraction of carotenoids that were quantified by measuring $A_{485}$.

Effect of growth phase and oxidative stress on heterologous expression of rpoE-lacZ fusion in $\boldsymbol{A}$. brasilense. In order to examine the effect of growth phase on induction of the Rsp rpoE promoter in $A$. brasilense, we performed $\beta$-galactosidase assays on exponential-phase as well as stationary-phase cells of $A$. brasilense strains harbouring pJDN30. To examine the effect of different reactive oxygen-species-producing chemicals, A. brasilense strains harbouring pJDN30 were exposed to oxidative stress by supplementing the midexponential-phase cultures with $2 \mathrm{mM} \mathrm{H}_{2} \mathrm{O}_{2}, 1 \mathrm{mM}$ paraquat or $10 \mu \mathrm{M}$ methylene blue and growing them for another $2 \mathrm{~h}$. Untreated cells were used as a control. At the time of assay, the number of cells in treated and control cultures was equalized by diluting them with fresh medium. $\beta$-Galactosidase assay (Miller, 1972) was performed 


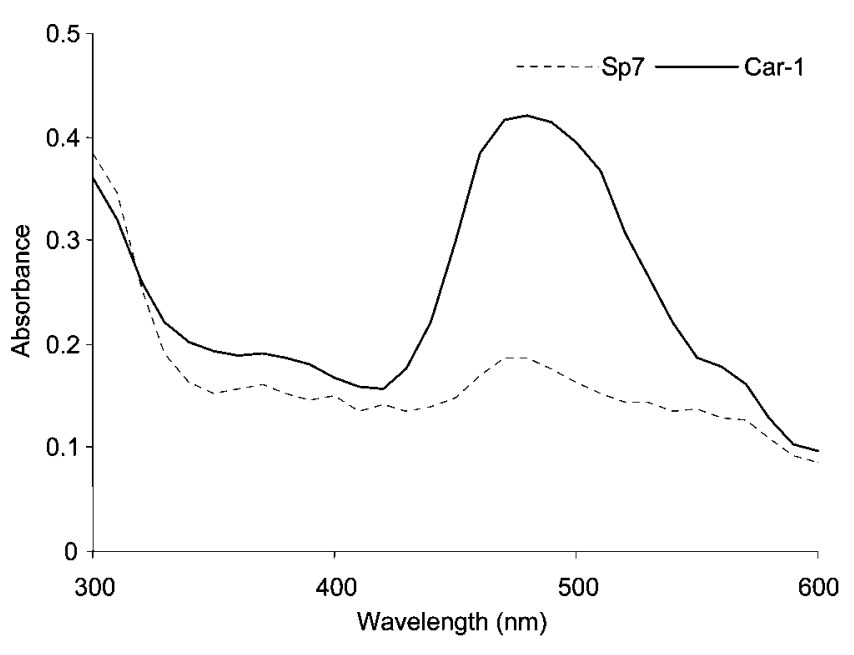

Fig. 1. Absorption spectra of methanol extracts prepared from $A$. brasilense Sp7 and its Car-1 mutant.

with $1 \mathrm{ml}$ equalized culture in triplicate for each sample on two different occasions.

\section{RESULTS}

\section{Isolation and identification of Tn5 mutants leading to carotenoid production in $A$. brasilense Sp7}

While screening a library of 6800 mutants of A. brasilense Sp7 generated by a mini-Tn 5 derivative containing a promoterless gusA reporter along with the oriV and nptII genes, on agar plates, two pink-coloured colonies representing mutants (designated Car-1 and Car-2) were observed. Expression of GusA was not observed in either mutant, possibly due to the insertion of Tn5 in the orientation wherein the promoterless gusA did not fuse with any promoter of $A$. brasilense. When absorption spectra of methanol extracts from the two Tn5 mutants were compared with their parent, they showed carotenoidspecific peaks (Fig. 1). It was also noted that aerobically grown cultures of the two Tn5 mutants (Car-1 and Car-2) produced carotenoids only during late-exponential phase to stationary phase, but not in early exponential phase (data not shown).

The genetic locus inactivated by $\operatorname{Tn} 5$ insertion in the two mutants (Car-1 and Car-2) was cloned, and the identity of the inactivated locus in each mutant was determined by nucleotide sequencing of genomic DNA flanking the site of Tn5 insertion. When plasmid pTN1 (harbouring the Tn5mutagenized genomic region from the Car-1 mutant) was electroporated into $A$. brasilense $\mathrm{Sp} 7$, the resulting transformants produced red-pigmented colonies. PCR amplification using primers specific for the chrR gene failed to produce any amplicon with the genomic DNA extracted from red-pigmented colonies, whereas an amplicon of $\sim 700 \mathrm{bp}$ was observed in the case of Sp7 genomic DNA. This confirmed that the chrR gene in the red-pigmented colonies was replaced by the Tn5-mutated chrR allele (data not shown). The deduced amino acid sequence of the ORF carrying the Tn5 insertion in $A$. brasilense Sp7 (231 aa) showed closest relatedness to the anti- $\sigma$ factor-encoding genes of $R$. rubrum (ZP_00267593, 224 aa) and M. magnetotacticum (ZP_00056407, 215 aa) (Supplementary Fig. S1). Among the other closely related anti- $\sigma$ factors, the deduced amino acid sequence of the putative anti- $\sigma$ factor from $A$. brasilense showed very high similarity ( $40 \%$ identity) with the ChrR protein of $R$. sphaeroides (ZP_00004392, 213 aa), which has been thoroughly investigated genetically, structurally and functionally (Campbell et al., 2007; Newman et al., 2001). In view of this, the anti- $\sigma$ factor protein of $A$. brasilense Sp7 was designated Abr ChrR. The sites of Tn 5 insertion in the Car-1 and Car-2 were 216 and 456 nt downstream of the start codon of the anti- $\sigma$ ORF, respectively (Fig. 2). Sequence analysis revealed that the Tn5 insertion was oriented such that the promoterless gusA could not be read from the promoter driving chrR, explaining the gus phenotype.

Primer walking on both sides of $\operatorname{Tn} 5$ revealed that the ORF of the anti- $\sigma$ was preceded by another ORF of 191 aa, which showed very high similarity to an ECF- $\sigma$ factor (COG1595), $\sigma^{\mathrm{E}}$ or RpoE (Fig. 2). The nucleotide sequences downstream of the anti- $\sigma$-encoding region revealed

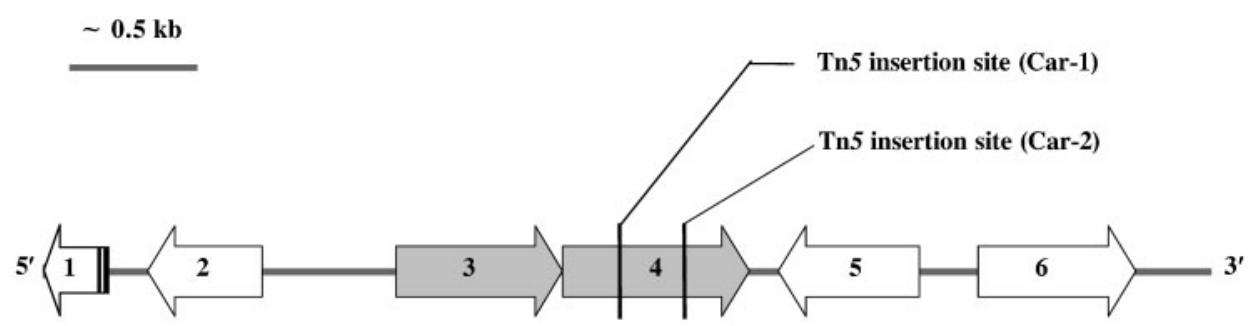

Fig. 2. Genetic map of the mutated locus involved in carotenoid overproduction in A. brasilense Sp7. Grey arrows represent identified ORFs and white arrows represent high-homology regions. ORFs: 1, Fe-S cluster redox enzyme; 2, hypothetical protein (ZP00268580); 3, ECF- $\sigma$ factor; 4, anti- $\sigma$ factor; 5, rRNA methylase; 6, L-asparaginase II. 
significant similarities with the genes encoding rRNA methylase and L-asparaginase II. Upstream of the ORF of ECF- $\sigma$, regions having significant similarities to a hypothetical protein of Nitrosomonas europea (ZP_00268580), followed by a gene encoding a redox enzyme containing a $\mathrm{Fe}-\mathrm{S}$ cluster, were identified.

Analysis of the nucleotide sequences of putative anti- $\sigma$ (named the chrR gene) and ECF- $\sigma^{\mathrm{E}}$ (named the $r p o E$ gene) region of $A$. brasilense revealed that both genes were very closely linked, to the extent that the start codon of the anti$\sigma$ ORF overlapped with the stop codon of the $\sigma^{\mathrm{E}} \mathrm{ORF}$, and thus they were probably transcribed together from a promoter located upstream of the ORF of $\sigma^{\mathrm{E}}$. The 191 amino acids of the putative ECF- $\sigma$ factor of A. brasilense showed maximum similarity ( $46 \%$ identity) to that of RpoE from R. sphaeroides (ZP_00004391, 181 aa; Fig. 3a), R. rubrum (ZP_00267592, 236 aa) and M. magnetotacticum (ZP_00056406, 225 aa) (Supplementary Fig. S2) which are located upstream of the ORF of their anti- $\sigma$ partners (COG3806). Interestingly, the single-nucleotide overlap of the start codon of the ORF of anti- $\sigma$ with the stop codon of the ORF of $\sigma$ in A. brasilense was also observed in the $R$. rubrum and M. magnetotacticum genomes. In $R$. sphaeroides, however, the ORFs of these two genes were distinct from each other without overlap of the start and stop codons.

Comparison of the translated ORF (191 aa) of the putative ECF- $\sigma$ factor of A. brasilense Sp7 revealed maximum similarity with $R$. sphaeroides in the whole of region 2, including 2.1, 2.2, 2.3 and 2.4, and in region 4.2. An examination of the ORF of anti- $\sigma$ of A. brasilense (Fig. 3b) revealed the presence of eight cysteine residues. In $R$. sphaeroides, ChrR possesses four cysteine residues, of which the two residues located at positions 35 and 38 at the Nterminal end constitute a conserved $\mathrm{Hx}_{3} \mathrm{Cx}_{2} \mathrm{C}$ zinc-binding domain, whereas the other two cysteine residues are located at positions 187 and 189 at the C-terminal end (Campbell et al., 2007; Newman et al., 2001). Both these cysteine-containing domains of ChrR in $R$. sphaeroides have been shown to be involved in interaction with $\sigma^{\mathrm{E}}$. Besides the two cysteines located at the 36th and 39th positions at the $\mathrm{N}$ terminal, which constitute the $\mathrm{Hx}_{3} \mathrm{Cx}_{2} \mathrm{C}$ zinc-binding domain in the ChrR of A. brasilense, the two other C-terminal cysteines also aligned well with positions

\section{(a) (ECF-sigma factor) \\ $R$, spaheroides MTDK------SDRTDWVALMRAIRDHRDEAAFAELFQHFAPKVKGFLMKSGSVASQAEEC 54 \\ A. brasilense MQDLGSPAAGADRT-FEELLTAVGRDRDRAAFAALFGHFAKRLKAYLRRQGCDAGGAEEL 59

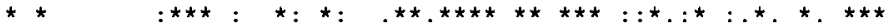

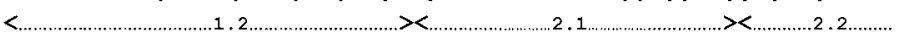 \\ R. Spaheroides AQDVMATVWQKAHLFDPSRASVATWIFTIARNRRIDGLRKDRQP--EPEDLFWGPDSEPD 112 \\ A. brasilense VQDVMLLVWRRAETYDPTQASAGTWVFTIARNRRIDALRREQRPEIDPADPTLVPDPMES 119

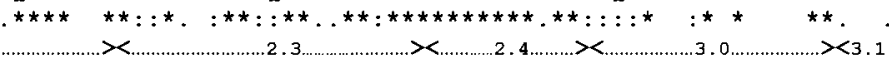 \\ R. spaheroides OADVYEMOOENARLGRAIARLPEAORALIERAFFGDLTHRELAAETGLPLGTIKSRIRLA 172 \\ A. brasilense ADDGVAARETAGRLRAALKTLPPEQADLLRLAYFEDKPHSLISAEQGIPLGTVKSRLRLA 179

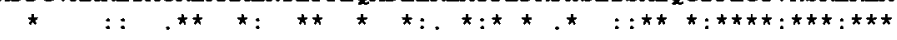

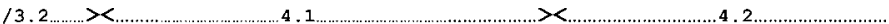 \\ R. spaheroides \\ LDRLRQHMS--- 181 \\ A. brasilense MERLRKAMRDSR 191

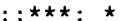

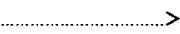

(b) (Anti-sigma factor)
R. sphaeroides
A. brasilense
R. sphaeroides
A. brasilense
R. sphaeroides
$A$. brasilense
R. sphaeroides

$A$. brasilense
MTIR-HHVSDALLTAYAAGTLSEAFSLVVATHLSLCDECRARAGALDAVGGSLMEETAPV 59 MTVPTHHPGDTLLIDYAGGALGEAASLIVATHLALCPCCRLNVAEMEAVGGALLESIEPE 60

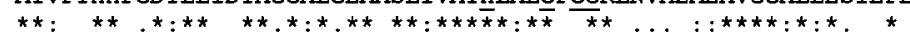
ALSEGSLASVMAQLDRQIQRPAPARRADPR-------APAPLADYVGRRLEDVRWRT 109 EVDPACLETVLAVLARLDDLPPLPRAPRPKLVCQPAEVPLLPEPLRRYVGSDLSRLPWKR 120

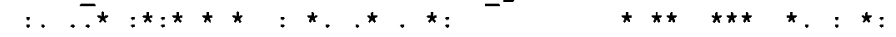
LGGGVRQAILPTGG--LMRGMDCYDIPLGSGSGYDSERRGKARIMRLASGVGPPHHTHRGIELTLVLDGGFTDDLG 180

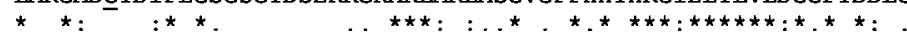
REGAGDIEIADQELEHTPVAERGLDCICLAATDAPLRFNSELPKIVQPFFRI 213 QFARGDLSVADDSVRHRPVADE-EGCLCCLAVTDAPLHFTGALGLILNPFVKF 231

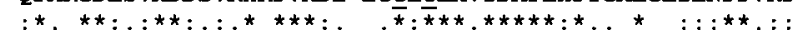

(c) Promoter

R. sphaeroides CITTTTCCCGCTCCCGCCTGATCCAGACTGGCCCGGCCGCGTAAGAGGACGTTAAGATCACGGC A. brasilense ATTTCGTATCGAAAGCCGGTGATCC GATGGCCCGCGGGTGCGTAGAAGA CCCATCGAACCTCAAGT

Fig. 3. (a) CLUSTAL $W$ alignment of the deduced amino acid sequence of $\sigma^{\mathrm{E}}$ of $A$. brasilense with that of $R$. sphaeroides. (b) CLUSTAL W alignment of the deduced amino acid sequence of anti- $\sigma^{\mathrm{E}}$ of $A$. brasilense with that of $R$. sphaeroides. (c) Comparison of $\sigma^{\mathrm{E}}$ promoter motifs (underlined) of $R$. sphaeroides with nucleotide sequences located in the $5^{\prime}$ untranslated region of the $\sigma^{\mathrm{E}}$ ORF in $A$. brasilense (underlined letters show similarity with the promoter motifs of $R$. sphaeroides). 
187 and 189 of the R. sphaeroides protein. In agreement with the high similarity of the ORFs of $\sigma^{\mathrm{E}}$ and anti- $\sigma^{\mathrm{E}}$ between $A$. brasilense and $R$. sphaeroides, when we compared the nucleotide sequences in the untranslated region upstream of the $\sigma^{\mathrm{E}}$, a typical $\sigma^{\mathrm{E}}$-dependent promoter structure of $R$. sphaeroides having -35 (TGATCC) and -10 (TAwww) regions separated by $18 \mathrm{nt}$ (Newman et al., 1999) was also present upstream of the ORF of $\sigma^{\mathrm{E}}$ in A. brasilense (Fig. 3c).

\section{Comparison of the nucleotide sequence of the rpoE and chrR genes and the size of anti-sigma factor protein from $A$. brasilense Sp7 and $A$. brasilense $\mathrm{Cd}$}

CLUSTAL w alignment of the 576 bp ORF of $r p o E$ from Sp7 and $\mathrm{Cd}$ showed that both the sequences were identical (data not shown). Analysis of the sequences of the chrR gene from $\mathrm{Sp} 7$ and Cd revealed that the size of the gene in Sp7 was $696 \mathrm{nt}$, while in Cd it was 695 nt. CLUSTAL W alignment of the $c h r R$ gene sequence from both the strains showed that nucleotide 466 of the chrR gene was missing in Cd (Fig. 4a). The occurrence of a run of four Cs around the point of deletion, as observed in this case, is normally a hotspot for frameshift mutations. A further analysis of the sequences downstream revealed that the deleted nucleotide changed the reading frame and resulted in the creation of a stop codon after seven amino acids. This is expected to reduce the size of anti- $\sigma$ factor from 231 aa in Sp7 to 162 aa in Cd.

The primers used for PCR amplification and cloning of the ORF of the $c h r R$ gene from Sp7 in pET15b were also used to amplify and clone the same gene from $\mathrm{Cd}$. When the proteins expressed from IPTG-induced cultures of E. coli BL21 DE3 (pLysS) carrying plasmids pAT3 (Sp7 sequence) and pAT4 (Cd sequence) were resolved by SDS-PAGE, it was observed that the overexpressed protein from pAT4 was about $7 \mathrm{kDa}$ smaller than that expressed from pAT3 (Fig. 4b). The His-tagged anti- $\sigma$ factor protein from Sp7 was expected to be of 249 aa $(\sim 27 \mathrm{kDa})$, whereas the truncated protein of $\mathrm{Cd}$ was expected to be of 183 aa $(\sim 20 \mathrm{kDa})$.

\section{Effect of expressing rpoE and chrR genes}

The rpoE and chrR genes, PCR-amplified from A. brasilense Sp7 genomic DNA, were cloned into the EcoRI and BamHI

(a)

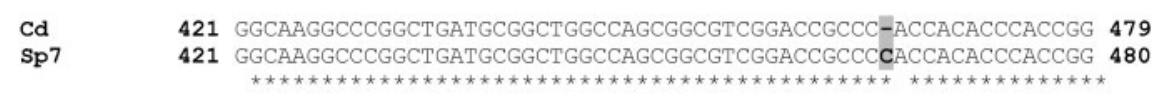

(b)

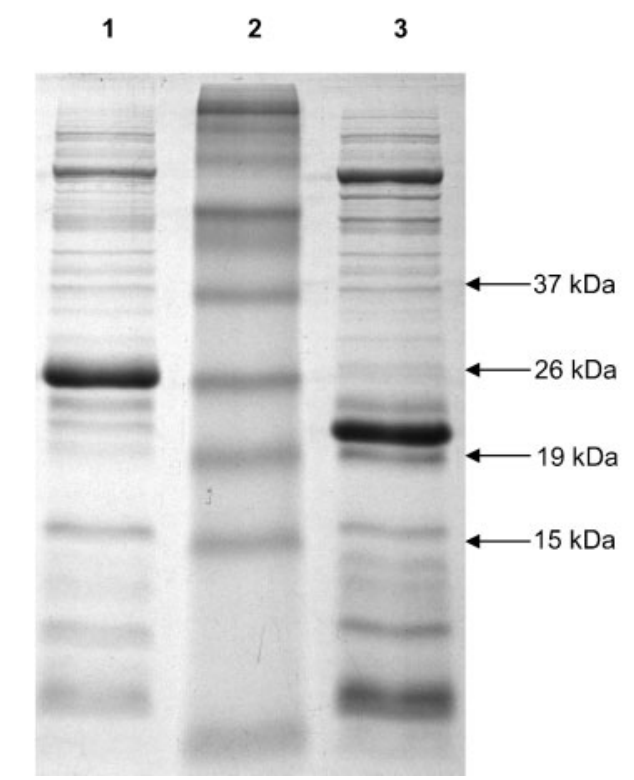

Fig. 4. (a) CLUSTAL $W$ alignment of the nucleotide sequence of the PCR-amplified chrR region from A. brasilense Sp7 and A. brasilense $\mathrm{Cd}$ showing a single deletion (highlighted in grey) at nucleotide 466 in $\mathrm{Cd}$. (b) SDS-PAGE of total proteins extracted from E. coli BL21 $\lambda$ DE3 (pLysS) harbouring plasmids pAT3 (lane 1) and pAT4 (lane 3). Lane 2 contains a molecular mass marker (BenchMark pre-stained protein ladder, Invitrogen). Indications of the molecular masses of relevant marker proteins are on the right of the figure. 
sites of the broad-host-range expression vector pMMB206, resulting in plasmids pAT5 and pAT6, respectively. Both these plasmids were transferred into E. coli S17-1 and conjugatively mobilized into two A. brasilense strains. A. brasilense Sp7 harbouring pAT5 showed slight orange-pink colouration indicating carotenoid synthesis (data not shown), but in the presence of $1 \mathrm{mM}$ IPTG, the cells produced an intense orange-pink colour showing overproduction of carotenoids. Wild-type A. brasilense Sp7 with or without IPTG did not produce carotenoids. On the other hand, A. brasilense Car-1 harbouring pAT6 displayed a slight orange-pink colour showing reduced synthesis of carotenoids compared with A. brasilense Car-1 (Supplementary Fig. S3). Addition of $1 \mathrm{mM}$ IPTG completely inhibited the production of carotenoids by $A$. brasilense Car-1 harbouring pAT6. Quantitative estimation of carotenoids also showed that when $r p o E$ was expressed in A. brasilense Sp7 via pAT5, the carotenoid content increased considerably. There was no increase in carotenoid content in A. brasilense Sp7 harbouring pMMB206 or pAT6 (expressing chrR). On the other hand, expression of rpoE via pAT5 showed very little increase in carotenoid content in the mutant Car-1. However, expression of $c h r R$ via pAT6 showed a considerable reduction of carotenoids in the Car-1 mutant (Fig. 5).

\section{Effect of growth phase and reactive oxygen species on heterologous expression of the rpoE $\mathbf{P} 1$ promoter of $\boldsymbol{R}$. sphaeroides in $A$. brasilense}

The strong sequence similarity of the ORFs of $\sigma^{\mathrm{E}}$ and anti$\sigma^{\mathrm{E}}$ of A. brasilense with those of $R$. sphaeroides, and also of

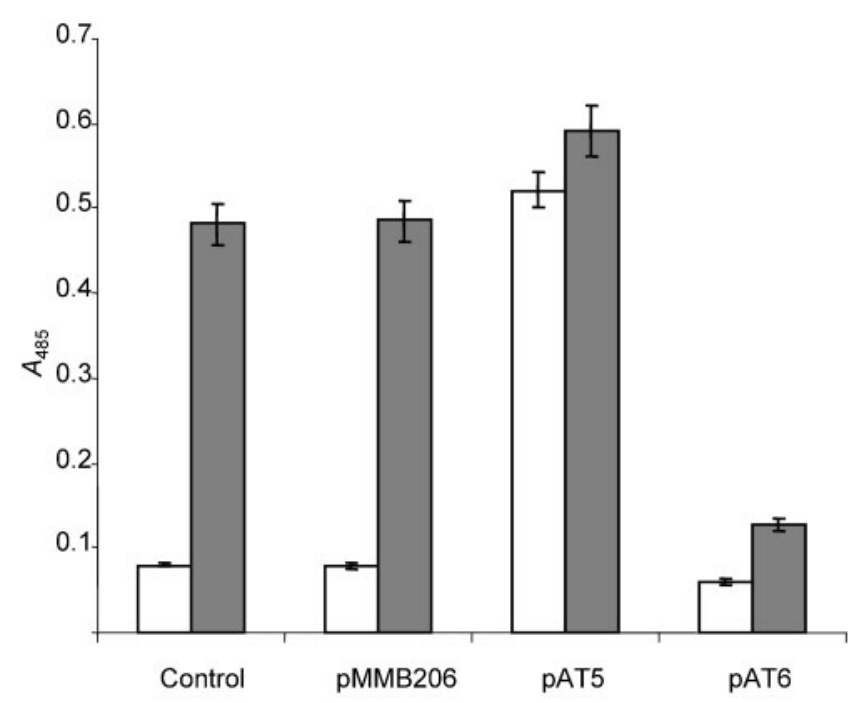

Fig. 5. Effect of $r p o E$ (via pAT5) and $c h r R$ (via pAT6) expression on carotenoid content in A. brasilense Sp7 (empty bars) and Car1 mutant (filled bars). Carotenoid content was determined by measuring $A_{485}$ of methanol extracts prepared from stationaryphase cultures grown with $1 \mathrm{mM}$ IPTG, as described in Methods. Error bars indicate SD from three replicates. the $r p o E \mathrm{P} 1$ promoter sequence with that present upstream of the ORF of $\sigma^{\mathrm{E}}$ in A. brasilense Sp7, prompted us to investigate whether the $\sigma^{\mathrm{E}}$-activated promoter of $R$. sphaeroides, rpoE P1, can be activated in A. brasilense. A transcriptional lac $Z$ fusion of $R$. sphaeroides rpoE $\mathrm{P} 1$ promoter in a broad-host-range vector was conjugatively mobilized into A. brasilense Sp7 and the Car-1 mutant. Assay of the $\beta$-galactosidase activity of the transconjugants showed that $R$. sphaeroides rpoE $\mathrm{P} 1$ was not activated in $A$. brasilense $\mathrm{Sp} 7$ but was activated in the Car-1 mutant, in which activation occurred during both exponential and stationary phases of growth (Fig. 6a). Interestingly, the expression of $R$. sphaeroides rpoE $\mathrm{P} 1$ promoter in the Car-1 mutant was more pronounced during stationary phase than in the exponential phase. When the effect of three different reactive oxygen species, superoxide, peroxide and singlet oxygen, on the heterologous expression of the Rsp $r p o E \mathrm{P} 1$ promoter was examined, paraquat did not show any effect, hydrogen peroxide showed very little increase, but methylene blue showed a pronounced increase in $\beta$ galactosidase activity (Fig. 6b).

\section{DISCUSSION}

Out of a pool of $6800 \mathrm{mTn} 5$ mutants of A. brasilense Sp7 we found two carotenoid-producing mutants. Both had insertions of mTn5 in chrR, the ORF encoding anti- $\sigma^{\mathrm{E}}$. This can be explained by hypothesizing that expression of the carotenoid biosynthetic genes in A. brasilense Sp7 is $\sigma^{\mathrm{E}}$ dependent (Anthony et al., 2005). Normally A. brasilense Sp7 has very low $\sigma^{\mathrm{E}}$ activity and low expression of its target genes (e.g. carotenoid-biosynthesis genes) because it has a functional anti- $\sigma^{\mathrm{E}}$. Inactivation of anti- $\sigma^{\mathrm{E}}$ in the two $\mathrm{mTn} 5$ mutants, Car-1 and Car-2, should activate $\sigma^{\mathrm{E}}$ and drive the expression of its target genes, resulting in the synthesis of significant levels of carotenoids. Consistent with this explanation, supplying intact $c h r R$ resulted in complete inhibition of carotenoid synthesis, and overexpression of $\sigma^{\mathrm{E}}$ resulted in carotenoid production. Analysis of the promoter regions of the carotenoid biosynthetic genes of the $A$. brasilense genome did not show the presence of the consensus $\sigma^{\mathrm{E}}$-dependent -35 sequence (TGATCC), indicating an indirect control of carotenoid biosynthetic genes by $\sigma^{\mathrm{E}}$.

These results led us to hypothesize that the naturally carotenoid-producing $A$. brasilense $\mathrm{Cd}$ strain might harbour mutation(s) in either rpoE or chrR. Indeed, the $c h r R$ gene in strain $\mathrm{Cd}$ had a single nucleotide deletion which resulted in a truncated protein (about $7 \mathrm{kDa}$ smaller than that of Sp7), whereas the rpoE gene sequence was identical in both the strains. In view of the earlier observations that the N-terminal 70-80 amino acid residues of the anti- $\sigma$ factor RseA and the first 85 amino acid residues of ChrR are sufficient to inhibit $\sigma^{\mathrm{E}}$ (Campbell et al., 2007), it might be expected that truncation at the Cterminal end would not inhibit the $\sigma^{\mathrm{E}}$-sequestering ability of Abr ChrR. However, in R. sphaeroides ChrR it has been 

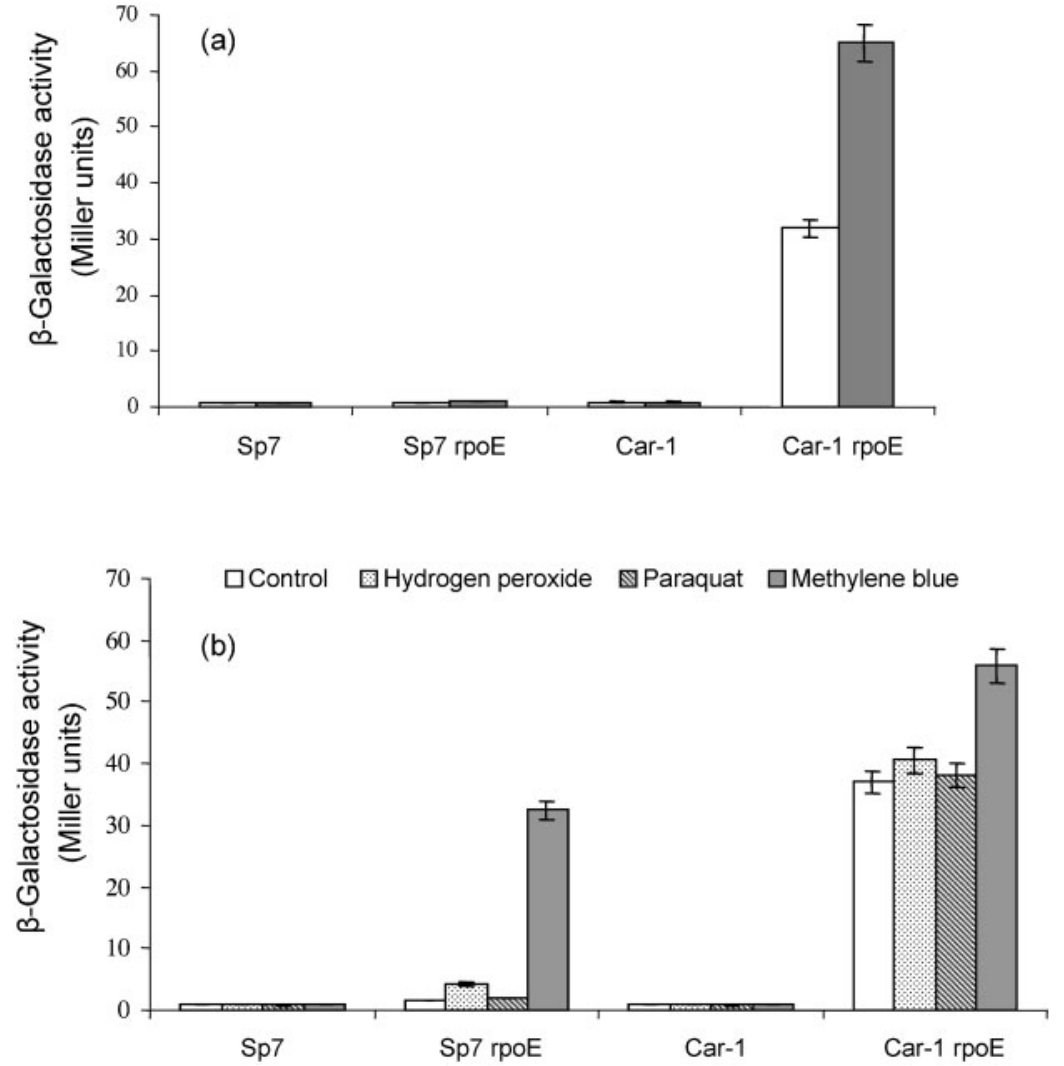

Fig. 6. (a). Heterologous expression of $R$. sphaeroides rpoE $\mathrm{P} 1$ ::lacZ transcriptional fusion ( $\mathrm{JJDN} 30$ ) in $A$. brasilense Sp7 and the Car-1 mutant during mid-exponential phase (empty bars) and stationary phase (filled bars). (b) Effect of hydrogen peroxide $(5 \mathrm{mM})$, paraquat $(1 \mathrm{mM})$ and methylene blue $(10 \mu \mathrm{M})$ on $\beta$-galactosidase activity of $\operatorname{Rsp} r p o E$ $\mathrm{P} 1$ : : lac $Z$ fusion. $\beta$-Galactosidase assays were performed three times in triplicate. Error bars indicate SD from three replicates. Sp7, empty wild-type strain; Sp7 rpoE, Sp7 carrying pJDN30; Car-1, empty mutant strain; Car-1 rpoE, Car-1 carrying pJDN30. shown clearly that replacement of the two cysteines located at positions 187 and 189 of ChrR with serine produces a mutant protein that fails to inhibit $\sigma^{\mathrm{E}}$ activity (Newman et al., 2001). Possibly, these two cysteines at the C-terminal end participate in maintaining the native structure of ChrR, and hence the truncated ChrR in the Cd strain may not be functional. These observations have clearly established that inactivation of the $c h r R$ gene by mutations (either by $\mathrm{Tn} 5$ insertion in the Car- 1 and Car 2 mutants or by a nucleotide deletion in strain $\mathrm{Cd}$ ) resulted in the carotenoid synthesis. The carotenoid-synthesizing phenotype was reversed by expressing the chrR gene in the Car-1 mutant as well as in A. brasilense $\mathrm{Cd}$.

The operon that encodes $\sigma^{\mathrm{E}}$ in A. brasilense is similar to that in $R$. sphaeroides. The $\sigma^{\mathrm{E}}$-dependent regulatory components of $A$. brasilense also showed strong similarity with those of $R$. sphaeroides. This included high similarity of the ORFs of $\sigma^{\mathrm{E}}$ and anti- $\sigma^{\mathrm{E}}$, similarity in the promoter structure and the occurrence of conserved cysteine residues in the ORF of anti- $\sigma^{\mathrm{E}}$ that are involved in interacting with $\sigma^{\mathrm{E}}$. The amino acid sequence of the $\sigma_{4}$ conserved domain of A. brasilense $\sigma^{\mathrm{E}}$ and the corresponding -35 promoter region showed $100 \%$ similarity to that of $R$. sphaeroides. On the other hand, the relatively lower degree of similarity in the -10 promoter sequence implied relatively lower similarity with the $\sigma_{2}$ domain of region 2 (Missiakas \& Raina, 1998). Conservation of the -35 sequence and its distance from the -10 sequence in $A$. brasilense and $R$. sphaeroides is important for promoter specificity and recognition by $\sigma^{\mathrm{E}}$ (Newman et al., 1999). The absence of similarity in the -10 sequences has been suggested to account for the co-existence of multiple members of the $\sigma^{\mathrm{E}}$ subfamily (Missiakas \& Raina, 1998). The anti- $\sigma^{\mathrm{E}}$ of $A$. brasilense Sp7 also showed similarity with the ChrR anti- $\sigma^{\mathrm{E}}$ of $R$. sphaeroides by lacking membrane-spanning domains and possessing a zinc binding domain $\left(\mathrm{Hx}_{3} \mathrm{Cx}_{2} \mathrm{C}\right)$ in the $\mathrm{N}$ terminal region. Recent structural studies of ChrR have revealed a two-domain architecture consisting of an $\mathrm{N}$ terminal anti- $\sigma$ domain (ASD) that sequesters $\sigma^{\mathrm{E}}$ by binding to $\mathrm{Zn}^{2+}$ in order to inhibit $\sigma^{\mathrm{E}}$-dependent transcription, and a C-terminal domain that adopts a cupin fold and coordinates an additional $\mathrm{Zn}^{2+}$ to carry out a transcriptional response (Campbell et al., 2007). The strong homology between the ChrR and anti- $\sigma$ of $A$. brasilense and presence of $\mathrm{Zn}^{2+}$ binding domains in the ASD clearly suggest that the anti- $\sigma$ belongs to the ZAS $\left(\mathrm{Zn}^{2+}\right.$ anti-sigma) subfamily of group IV anti-sigmas.

Although ORFs of $\sigma^{\mathrm{E}}$ and anti- $\sigma^{\mathrm{E}}$ in A. brasilense showed maximum similarity with those of $R$. sphaeroides, $\sigma^{\mathrm{E}}$ of $R$. sphaeroides does not appear to directly control the biosynthetic genes of carotenoids as they lack $\sigma^{\mathrm{E}}$-dependent promoters (Anthony et al., 2005). It has been clearly established that the rpoE $\mathrm{P} 1$ promoter in R. sphaeroides is $\sigma^{\mathrm{E}}$ dependent. In view of the strong similarity of the $\sigma^{\mathrm{E}}$ and of the putative promoter sequences located in the untranslated region of $\sigma^{\mathrm{E}}$ of $A$. brasilense with those of $R$. sphaeroides, it 
was expected that $\sigma^{\mathrm{E}}$-dependent promoters of R. sphaeroides should be activated in A. brasilense as well. Activation of the $\sigma^{\mathrm{E}}$-activated promoter, rpoE $\mathrm{P} 1$ of $R$. sphaeroides in the Car1 mutant confirmed that the rpoE $\mathrm{P} 1$ promoter can indeed be identified and activated in vivo by the free $\sigma^{\mathrm{E}}$. Carotenoid biosynthesis has earlier been shown to be regulated by ECF $\sigma$ factor in the Gram-negative bacterium Myxococcus xanthus (Browning et al., 2003) and some Gram-positive bacteria belonging to the genus Streptomyces (Takano et al., 2005). In M. xanthus, carotenoid synthesis is regulated in response to copper and blue light; the latter is sensed by a membranebound anti- $\sigma$ factor, CarR, followed by the transduction of the signal to the ECF $\sigma$ factor CarQ, which in turn drives the expression of the carQRS operon and of the crtI gene, depending upon exposure to light and dark conditions (Browning et al., 2003). The synthesis of carotenoids in the Gram-positive genus Streptomyces, however, may be constitutive, light-dependent or cryptic. Earlier genetic studies of carotenoid production in two species of Streptomyces, Streptomyces setonii (Kato et al., 1995) and Streptomyces griseus (Schumann et al., 1996), have shown that carotenogenesis is cryptic. Carotenoid production in these two species is induced by an increased copy number of a gene that encodes a stress-response $\sigma$ factor, $\sigma^{\mathrm{CrtS}}$, suggesting the involvement of this $\sigma$ factor in the transcription of carotenoid biosynthetic genes. On the other hand, Streptomyces coelicolor A3 does not have a crtS homologue in its genome, and its light-induced carotenoid synthesis is under the control of an ECF $\sigma$ factor, $\sigma^{\text {LitS }}$ (Takano et al., 2005).

We observed that A. brasilense Sp7 did not show any notable $\sigma^{\mathrm{E}}$ activity during exponential or stationary phase, but when anti- $\sigma^{\mathrm{E}}$ was mutated the mutant showed notable $\sigma^{\mathrm{E}}$ activity during exponential phase, which increased further during stationary phase. This result is similar to that in E. coli, where $\sigma^{\mathrm{E}}$ is activated upon entry into stationary phase, due to starvation, by the alarmone ppGpp, even if its cognate anti- $\sigma$ factor is inactivated (Costanzo \& Ades, 2006; Costanzo et al., 2008). Therefore, stationary phase could be one of the conditions during which $r p o E$ is activated only in anti- $\sigma$ mutants of $A$. brasilense. $\sigma^{\mathrm{E}}$ and anti- $\sigma^{\mathrm{E}}$ of $A$. brasilense Sp7 showed maximum similarity to $\sigma^{\mathrm{E}}$ and $\mathrm{ChrR}$ of $R$. sphaeroides, respectively, and their regulatory behaviour was also similar. As observed in $R$. sphaeroides (Anthony et al., 2005), methylene blue induced $\sigma^{\mathrm{E}}$ activity, whereas hydrogen peroxide and paraquat did not. Interestingly, in A. brasilense methylene blue increased the $\sigma^{\mathrm{E}}$ activity substantially, whether or not an anti- $\sigma$ was present. Thus, out of the two well-studied zinc anti- $\sigma$ factors, ChrR of $R$. sphaeroides and RsrA of $S$. coelicolor, the A. brasilense anti- $\sigma$ factor is similar to RsrA in having seven cysteines, but its ability to respond to methylene blue and not to paraquat or hydrogen peroxide is more like that of ChrR of $R$. sphaeroides (Campbell et al., 2007).

In this report we have clearly established that the inactivation of anti- $\sigma^{\mathrm{E}}$ in A. brasilense leads to carotenoid synthesis in a
$\sigma^{\mathrm{E}}$-dependent manner. The operon that includes the $r p o E$ gene in A. brasilense is bicistronic and similar to that of $R$. sphaeroides. However, carotenoid synthesis in M. xanthus and Streptomyces species is controlled by rpoE, which is part of a polycistronic operon and controlled by light and copper. Since none of the carotenoid biosynthetic genes contained a consensus $\sigma^{\mathrm{E}}$-dependent promoter, it is expected that $\sigma^{\mathrm{E}}$ controls carotenoid synthesis indirectly through intermediate regulators. We are currently investigating the environmental conditions/signals that can induce carotenoid synthesis and the alternative $\sigma$ factors that might be involved in $\sigma^{\mathrm{E}}$ dependent control of carotenoid biosynthesis in A. brasilense.

\section{ACKNOWLEDGEMENTS}

This work was partly supported by a grant from the Indo-French Centre for Promotion of Advanced Research, New Delhi, sanctioned to A.K.T., an Overseas Associateship by the Department of Biotechnology, Government of India, to A.K.T. Grant \# 5RO1GM036278 to C.A. G. and an ASM-UNESCO travel grant to N.T. are gratefully acknowledged. We thank Igor Jouline (Oak Ridge National Laboratory) for providing access to the partial genome sequence of $A$. brasilense, Timothy Donohue (University of Wisconsin-Madison) for suggestions and for providing pJDN30, Anne Van Dommelen (KU-Leuven) for valuable suggestions, and the Council of Scientific and Industrial Research, New Delhi, for a senior research fellowship for N.T.

\section{REFERENCES}

Abdel-Salam, M. S. \& Klingmueller, W. (1987). Transposon Tn5 mutagenesis in Azospirillum lipoferum: isolation of indole acetic acid mutants. Mol Gen Genet 210, 165-170.

Altschul, F. F., Madden, T. L., Schäffer, A. A., Zhang, J., Zhang, Z., Miller, W. \& Lipman, D. J. (1997). Gapped BLAST and PSI-BLAST: a new generation of protein database search programs. Nucleic Acids Res 25, 3389-3402.

Anthony, J. R., Warczak, K. L. \& Donohue, T. (2005). A transcriptional response to singlet oxygen, a toxic byproduct of photosynthesis. Proc Natl Acad Sci U S A 102, 6502-6507.

Browning, D. F., Whitworth, D. E. \& Hodgson, D. A. (2003). Lightinduced carotenogenesis in Myxococcus xanthus: functional characterization of the ECF sigma factor CarQ and anti-sigma factor CarR. Mol Microbiol 48, 237-251.

Campbell, E. A., Greenwell, R., Anthony, J. R., Wang, S., Lionel, L., Das, K., Sofia, H. J., Donohue, T. J. \& Darst, S. A. (2007). A conserved structural module regulates transcriptional responses to diverse stress signals in bacteria. Mol Cell 27, 793-805.

Costanzo, A. \& Ades, S. E. (2006). Growth phase-dependent regulation of the extracytoplasmic stress factor, $\sigma^{\mathrm{E}}$, by guanosine 3',5'-bispyrophosphate (ppGpp). J Bacteriol 188, 4627-4634.

Costanzo, A., Nicoloff, H., Barchinger, S. E., Banta, A. B., Gourse, R. L. \& Ades, S. E. (2008). ppGpp and DksA likely regulate the activity of the extracytoplasmic stress factor $\sigma^{\mathrm{E}}$ in $E$. coli by both direct and indirect mechanisms. Mol Microbiol 67, 619-632.

D'Souza, S. E., Altekar, W. \& D'Souza, S. F. (1997). Adaptive response of Haloferax mediterranei to low concentrations of $\mathrm{NaCl}(<20 \%)$ in the growth medium. Arch Microbiol 168, 68-71.

Fong, N. J. C., Burgess, M. L., Barrow, K. D. \& Glenn, D. R. (2001). Carotenoid accumulation in the psychrotrophic bacterium 
Arthrobacter agilis in response to thermal and salt stress. Appl Microbiol Biotechnol 56, 750-756.

Hartmann, A. \& Hurek, T. (1988). Effect of carotenoid overproduction on oxygen tolerance of nitrogen fixation in Azospirillum brasilense Sp7. J Gen Microbiol 134, 2449-2455.

Jagannadham, M. V., Rao, V. J. \& Shivaji, S. (1991). The major carotenoid pigment of a psychrotrophic Micrococcus roseus strain: purification, structure, and interaction with synthetic membranes. J Bacteriol 173, 7911-7917.

Kato, F., Hino, T., Nakaji, A., Tanaka, M. \& Koyama, Y. (1995), Carotenoid synthesis in Streptomyces setonii ISP5395 is induced by the gene $\mathrm{crtS}$, whose product is similar to a sigma factor. Mol Gen Genet 247, 387-390.

Kumar, S., Tamura, K. \& Nei, M. (2004). MEGA3: integrated software for molecular evolutionary genetics analysis and sequence alignment. Brief Bioinform 5, 150-163.

Mehnaz, S., Weselowski, B. \& Laza, G. (2007). Azospirillum canadense sp. nov., a nitrogen-fixing bacterium isolated from corn rhizosphere. Int J Syst Evol Microbiol 57, 620-624.

Miller, J. H. (1972). Experiments in Molecular Genetics. Cold Spring Harbor, NY: Cold Spring Harbor Laboratory.

Missiakas, D. \& Raina, S. (1998). The extracytoplasmic function sigma factors: role and regulation. Mol Microbiol 28, 1059-1066.

Morales, V. M., Backman, A. \& Bagdasarian, M. (1991). A series of wide-host-range low copy-number vectors that allow direct screening for recombinants. Gene 97, 39-47.

Nagarajan, T., Vanderleyden, J. \& Tripathi, A. K. (2007). Identification of salt stress inducible genes that control cell envelope related functions in Azospirillum brasilense Sp7. Mol Genet Genomics 278, 43-51.

Newman, J. D., Falkowski, M. J., Schilke, B. A., Anthony, L. C. \& Donohue, T. J. (1999). The Rhodobacter sphaeroides ECF sigma factor, $\sigma^{\mathrm{E}}$, and the target promoters cycA P3 and rpoE P1. J Mol Biol 294, 307-320.

Newman, J. D., Anthony, L. C. \& Donohue, T. J. (2001). The importance of zinc-binding to the function of Rhodobacter sphaeroides ChrR as an anti-sigma factor. J Mol Biol 313, 485-499.

Nur, I., Yuval, L. S., Okon, Y. \& Henis, Y. (1981). Carotenoid composition and function in nitrogen-fixing bacteria of the genus Azospirillum. J Gen Microbiol 122, 27-32.
Nur, I., Okon, Y. \& Henis, Y. (1982). Effect of dissolved oxygen tension on production of carotenoids, poly- $\beta$-hydroxybutyrate, succinate oxidase and superoxide dismutase by Azospirillum brasilense Cd grown in continuous culture. J Gen Microbiol 128, 2937-2943.

Saitou, N. \& Nei, M. (1987). The neighbor-joining method: a new method for reconstructing phylogenetic trees. Mol Biol Evol 4, 406-425.

Schumann, G., Nurnberger, H., Sandmann, G. \& Krugel, H. (1996). Activation and analysis of cryptic crt genes for carotenoid biosynthesis from Streptomyces griseus. Mol Gen Genet 252, 658-666.

Steenhoudt, O. \& Vanderleyden, J. (2000). Azospirillum, a free-living nitrogen-fixing bacterium closely associated with grasses: genetic, biochemical and ecological aspects. FEMS Microbiol Rev 24, 487-506.

Takano, H., Obitsu, S., Beppu, T. \& Ueda, K. (2005). Light-induced carotenogenesis in Streptomyces coelicolor A3 (2): identification of an extra-cytoplasmic function sigma factor that directs photodependent transcription of the carotenoid biosynthesis gene cluster. J Bacteriol 187, 1825-1832.

Tarrand, J. J., Krieg, N. R. \& Dobereiner, J. (1978). A taxonomic study of the Spirillum lipoferum group, with descriptions of a new genus, Azospirillum gen. nov. and two species, Azospirillum lipoferum (Beijerinck) comb. nov. and Azospirillum brasilense sp. nov. Can J Microbiol 24, 967-980.

Thompson, J. D., Higgins, D. G. \& Gibson, T. J. (1994). CLUSTAL W: improving the sensitivity of progressive multiple sequence alignment through sequence weighting, position-specific gap penalties and weight matrix choice. Nucleic Acids Res 22, 4673-4680.

Vanstockem, M., Michiels, K., Vanderleyden, J. \& Van Gool, A. (1987). Transposon mutagenesis of Azospirillum brasilense and Azospirillum lipoferum. Physical analysis of Tn5 and Tn5-mob insertion mutants. Appl Environ Microbiol 53, 410-415.

Wisniewska, A. \& Subczynski, W. K. (1998). Effects of polar carotenoids on the shape of the hydrophobic barrier of phospholipid bilayers. Biochim Biophys Acta 1368, 235-246.

Woese, C. R., Blanz, P., Hespell, R. B. \& Hahn, C. M. (1982). Phylogenetic relationship among various helical bacteria. Curr Microbiol 7, 119-124.

Edited by: H.-M. Fischer 